\title{
Research and Development of Fire Fighting Facilities Management System based on .Net
}

\author{
Jiyuan Feng ${ }^{1, a}$, Shengbin Zhao ${ }^{2, b^{*}}$ and Chong $\mathrm{Xu}^{3, \mathrm{c}}$ \\ 1 School of Information \& Control engineering, Shenyang Jianzhu University, Shenyang, China

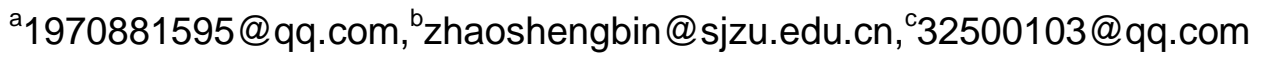 \\ ${ }^{*}$ The Corresponding author
}

\section{Keywords: Fire facilities; ArcGIS; Management system; WebGIS; B/S mode}

\begin{abstract}
Objective: In order to effectively promote rural fire control facilities and emergency management ability, the firefighting facilities management system uses the ArcGIS and SQL Server, and adopts $\mathrm{B} / \mathrm{S}$ pattern design. It can organize combination of spatial data and quantitative analysis. This system has realized system management functions such as the basic information, the dangerous source, fire control facilities management, and fire disaster analysis, the rural fire control facilities spatial database is set up, and provides the dynamic intuitive way to simulate fire occurrence. Methods: It provides a digital platform for fire control facilities management, provides the technical support for fire planning and emergency decision-making, Results: it has the advantages of strong interaction, convenient operation, and expansion ability as well. Conclusion: The system will greatly promote the modern management methods of fire prevention, fire fight and application value works in our country.
\end{abstract}

\section{Introduction}

At present the trend of the development of the cities and towns of our country is conditioned by the geographical location and the historical conditions, disaster tolerance is very limited, and scientific management of disaster prevention facilities started late, the various disaster prevention infrastructure is weakness. Management related to the disaster management agency lacks the disaster relief system of unified coordination mechanism, cannot form of disaster preparedness and response events forces. Along with our country urbanization construction has made more money, will need to set up high-tech new theoretical guidance of modern disaster management mode, solve the weakness in front of the disaster situation, this is the direct cause of this system development.

Existing computer simulation techniques in general only simulate fire information. For fire emergency plan should not only consider the fire, combining the infrastructure and the distribution of hazard is also necessary. Using $\mathrm{B} / \mathrm{S}$ mode which is easy to expand, strong sharing, and simple to maintain and so on is a good way to solve the problem. Using B/S mode to achieve the general data maintenance personnel and disaster policy makers, from a different location, with different way of access, access and manipulate a common spatial data. Villages and towns is in accordance with the geographical distribution of the characteristics of $\mathrm{B} / \mathrm{S}$ mode function, as long as there is browser terminal connected to the Internet, can operate on infrastructure and disaster relief decisions. Based on the .Net development system, through different channels to collect area picture, coordinates, and all kinds of attribute data and spatial data stored in the data layer on the server, put the business processing of the data on the middle business layer, by applying system developed by this model to complete the disaster simulation and measurement, to ensure the integrity of the structure of system development and efficiency.

The author adopts B/S pattern design, in the information management of fire control facilities and hazards of villages and towns on the basis of above, to carry on the organic combination and quantitative comprehensive analysis of the space data, making rapid fire analysis and simulation, and provides a reliable support for quickly handling fire prediction and emergency rescue development. 


\section{The Related Work of Disaster Prevention System}

Research institutions in the western country, apply advanced information technology research achievement such as the geographic information system (GIS), global positioning system (GPS), remote sensing (RS), to modern network computing technology disasters prevention and treatment process early. Compared with the traditional meaning of the geographic information system, WebGIS technology is the developing direction of modern computer technology. [1] The information in the GIS mainly need of spatial data in graphics, image way, through the human-computer interaction to query spatial data analysis, and so on. Applying geographic information request/response mechanism of the HTTP protocol, form the transmission on the Internet, and displayed on the browser WebGIS has more extensive use of multimedia data. WebGIS, based on the network client/server system, is to use the Internet for the exchange of information between the client and the server, this means that the transmission of information can be in any place, and the system is a distributed system, the user and the server can be distributed in different locations and different computer platforms.

When choice the rural fire control facilities management information system on WebGIS platform, on the principle of light workload, light maintenance and light memory, detailed consider factors such as applications range, the system research and development of technical requirements, the software system technical support and user's economic receptivity and so on, not only have the function of object-oriented, and also have the development of high efficiency, good compatibility, good portability and order running speed, etc. In our country rural disaster prevention disaster relief work, using WebGIS technology will greatly improve the work efficiency. [2]

\section{Design based on the .Net System}

Functional Structure Design. According to the characteristics of the rural fire control facilities management system, puts forward to establish basic information, hazards source, fire prevention facility management module and the analysis of the fire control module and system architecture, through the establishment of the spatial database in the fire control facilities, the fusion of disaster digital simulation and public safety emergency management, provide information support for planning and decision-making, provide fire facilities information management, emergency decision digital platform for the management department. [3] The function structure of villages and towns fire control facilities management information system based on.net is shown in fig. 1.

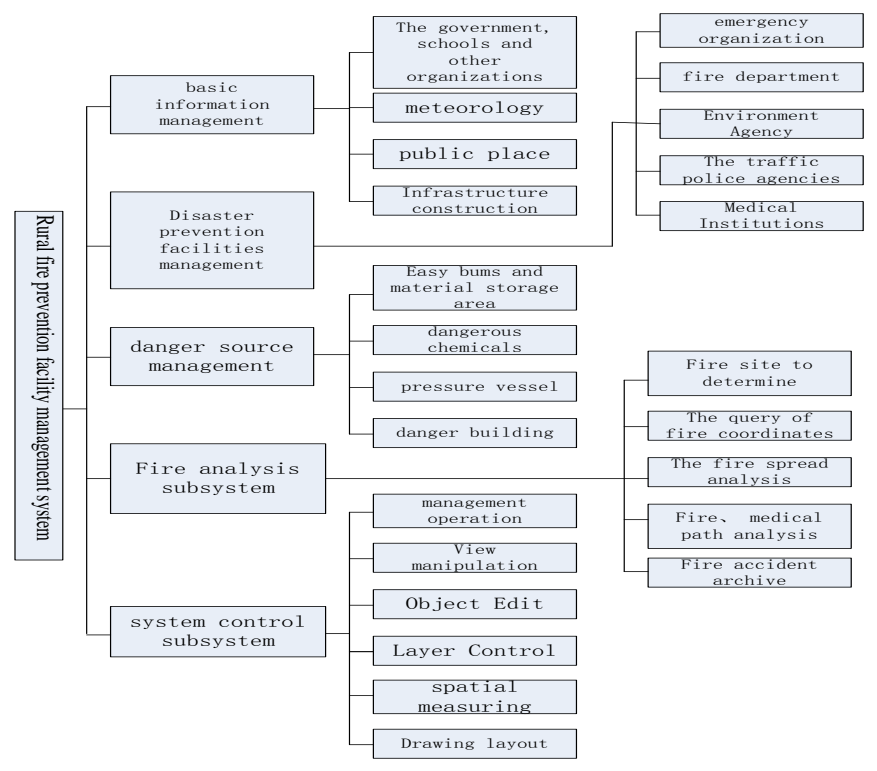

Figure 1. Finite Systematic Function Structure

The Architecture Design. Rural fire control facilities management information system use the multi-layer architecture based on $\mathrm{B} / \mathrm{S}$ mode, to provide information management system which can 
be operated on the Internet. The whole system structure is divided into customer layer, service layer and data layer, [4] as shown in fig. 2.

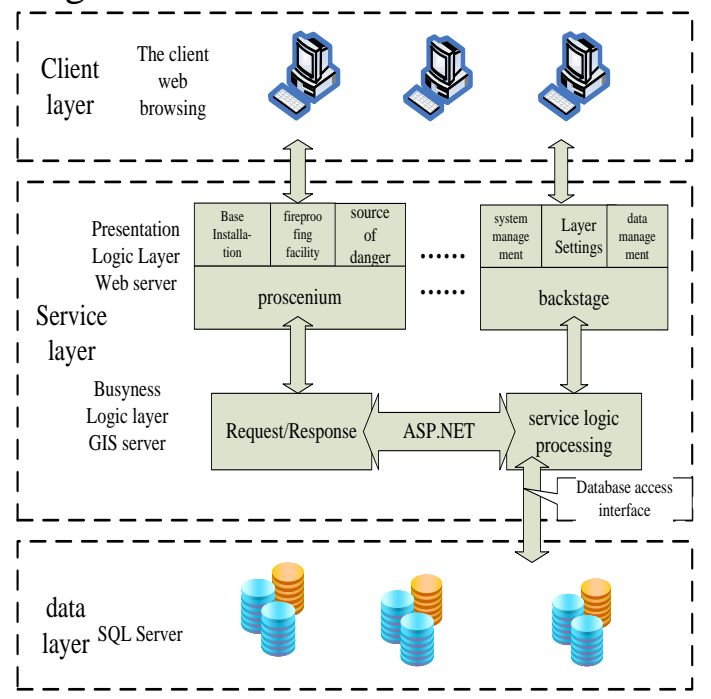

Figure 2. Finite Architecture Based on B/S

Customer layer is responsible for the various requests and system response of users, its business includes different user input and output interface display, it passes the user requests and Server responses between the Web servers of Presentation logic layer based on the HTTP protocol. The service layer is Web Server gateway between the client and the GIS Server and database Server, Responsible for Web access control service; handle includes user base information, fire control facilities and hazard management, systems and control, control operation parameters, such as data transfer and business processing results back down, etc. [5]The GIS Server is the core of the whole system business which can be independent, also can be integrated with the Web Server, responsible for the processing of spatial data, spatial data access, map display, spatial data analysis and other services. Data layer is responsible for handling service request and response through the database access interface, its business includes database organization, user information management, basic information query, spatial data analysis and map information management services.

Access to Data Model and Interface Design. Rural fire control facilities management information system is mainly including buildings, hazards, infrastructure and other attribute data and spatial data. Safe operate on data through the data access interface for data for.

Spatial Data Source. Area of spatial data sources have a lot of classes, including RS images, planning and design, CAD drawings, GPS data, and various kinds of social and economic statistical data, the remote sensing image is to obtain and update the new methods of spatial data, and can provide real-time, accurate, comprehensive information and a wide range of resources and environment. By using RS image stitching, interpretation, using the existing engineering data or GPS measurement data, after handling the RS image registration, as the basis of spatial database building library. [6, 7]

The Conceptual Model. The system based on a spatial attribute database data operation result accuracy, with security, stability, maintainability and extensibility for the principle, conform to the actual demand of spatial database is established. For different map of dot, line, face, etc. each spatial data storage, will be designed the corresponding attribute database, as shown in fig. 3 . 


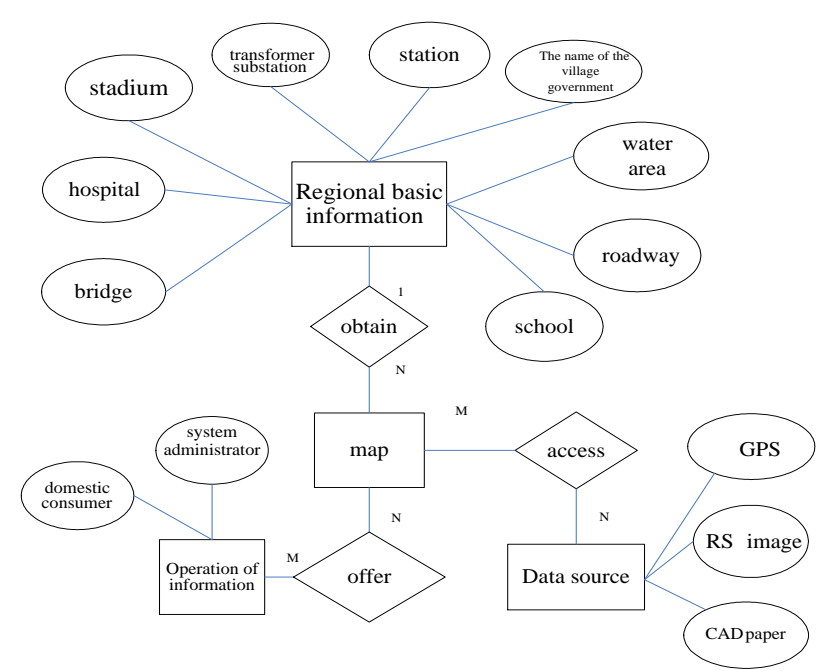

Figure 3. Finite E-R Diagram of Base Data

The Logical Model. Based on the principle of software system design specification and the data model design, logic design including rural fire control facilities management information system building facilities, infrastructure, and disaster data model design and disaster analysis data model design two aspects of content. The logic building information model of the system is shown in table 1 .

Table 1 Buildings basis information

\begin{tabular}{|c|c|c|}
\hline name & code & Data type \\
\hline order & ID & varchar(20) \\
\hline area of structure & area & decimal(20, 6) \\
\hline number of people & Population & int \\
\hline phone & Phone & varchar(20) \\
\hline Layer number & Layers & int \\
\hline Layer hight & LayerHeight & decimal(5, 2) \\
\hline usage & Uses & varchar(100) \\
\hline building structure & Architecture & int \\
\hline buildtime & Constructiontime & datetime \\
\hline The disaster level & QualityLevel & int \\
\hline UERDI & EarthquakeIndex & int \\
\hline building zone & BuildingWorth & decimal(20, 6) \\
\hline service area & PropertyWorth & decimal(20, 6) \\
\hline
\end{tabular}

Data Access Interface. System is through the database access interface implementation of each module of the database calls and control. To achieve data sharing and coordination work, fire analysis subsystem set fire in the system analysis model parameters and model operation step by step, parameter is set into variables, by analysis model from the variable model runtime reads the parameters, return results provide the basis for decision-making[8-13], as shown in fig. 4.

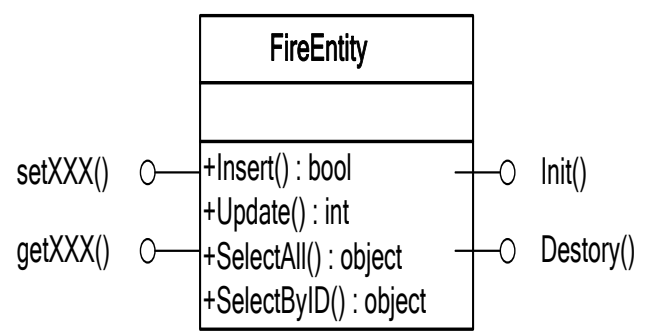

Figure 4. Finite Data Access Interface of Fire Entity 
Tasks of other query calls directly from spatial database read the related data or attribute data, then transfer to the calling task. Ensure the operating flexibility and the security of the database.

\section{Conclusion}

Based on the .net fire control facilities management system in rural areas is a more complicated and has a developmental systems engineering, need more discipline, more technical to support. The system applies general data access and processing technology, can support different data sources, including SQL Server, thus the system has stronger practical, and has better security and faster response speed, improved the service quality and the applicability of the system.

Rural fire control facilities management information system for the purpose of preventing fire, Will greatly promote the modern management methods of fire prevention and fire fighting work in our country, by integrating rural fire information data and the integrated application of fire power, To improve the quality of rural population in China life, promote the development of urbanization construction development with infinite space.

\section{References}

[1] Zhou Quan, Analysis of influencing factors of emergency management for natural disasters[D].Wuhan: Huazhong University Of Science And Technology,2010.

[2] Marta Yebra, Philip E. Dennison, Emilio Chuvieco, David Ria? o, Philip Zylstra, E. Raymond Hunt, F. Mark Danson, Yi Qi, Sara Jurdao. A global review of remote sensing of live fuel moisture content for fire danger assessment: Moving towards operational products [J]. Remote Sensing of Environment . 2013

[3] Xin Jing,Huang Chongfu, Mechanisms and probability model of buildings fire spread [J]. Fire Science and Technology,2011,10: 886-888.

[4] Cao Min, Huang Jihai. The Implementation and application of construction safety supervision and management system based on Web server[C]. Paris, France: The 2nd International Conference on Computer Application and System Modeling, 2012.

[5] Liu Wenquan. Framework and application of decision support system for oil spill emergency on offshore platform based on GIS[D].Qingdao: Ocean University of China,2010.

[6] Jose Raul Romo Leon, Willem J. D. van Leeuwen, Grant M. Casady. Using MODIS-NDVI for the Modeling of Post-Wildfire Vegetation Response as a Function of Environmental Conditions and Pre-Fire Restoration Treatments. Remote Sensing of Environment . 2012

[7] He Junliang, Zheng Yanxia. Design of non-full irrigation management information system of hebei province based on GIS[M].Berlin Heidelberg: Computer and Computing Technologies in Agriculture IV,2011.

[8] Tong Dawei, Sun Yichao. Comprehensive risk analysis of storm surge flood based on Web GIS[J]. Transactions of Tianjin University,2012(18):194-200.

[9] Zhao Liyuan. Microsimulation of integrated land use and transportation based On GIS[D].Chengdu: Southwest Jiaotong University,2011.

[10]Test Bed Design of Fire Detection System Based on Multi-Sensor Information for Reduction of False Alarms Lee, G.J.; Kim, H.G.; Lee, B.U.; Kim, T.O.; Sin, D.I. Source: Journal of The korean Institute of GAS, v 16, n 6, PAGERANGE:107, 2012.

[11] The technical proposal to reduce the fire false alarm for the automatic fire alarm system Choi, G.C. Source: Korea Fire Institute Research Report, PAGERANGE: 141-146, 2015 
[12]Development of Smart Phone Applications Linked with Fire Alarm Control Panel in Automatic Fire Detection System Kong, H.S.; Yang, J.S.; Kang, K.S. Source: International Journal of Smart Home, v 10, n 2, PAGERANGE:11-18, 2016.

[13] Yang Chanjian. Numerical simulation on billboards fire of commercial building [J].Fire Science and Technology, 2011 (30):701-704. 